\title{
Training Schedule and Sleep in Adolescent Swimmers
}

\author{
Sigridur L. Gudmundsdottir \\ University of Iceland
}

\begin{abstract}
Purpose: Insufficient sleep duration may affect athletic performance and health. Inconsistent sleep pattern also has negative health effects, but studies on athletes' intraindividual sleep variability are scarce. The aim of this research was to compare total sleep time (TST) and variability (TST-variability), wakening after sleep onset, and sleep efficiency, during nights preceding early morning practices with other nights, and to investigate sleep characteristics of nights following a day with early morning only, evening only, or both a morning and an evening session in adolescent swimmers. Methods: Wrist-worn accelerometers were used to measure 1 week of sleep in 108 swimmers (mean age 16.1 [2.6] y) in Iceland. Adjusted regression analyses and linear mixed models were used to explore associations of training schedules with TST, TST-variability, wakening after sleep onset, and sleep efficiency. Results: Mean TST was 6:32 (h:min) ( $\pm 39 \mathrm{~min})$ and TST-variability was 63 minutes $( \pm 25$ min). TST decreased and TST-variability increased with more early morning practices. TST preceding early training was 5:36 and 5:06 in $<16-$ and $\geq 16-$ year-olds, respectively, shorter than on nights preceding later or no morning training $(P<.001)$. Conclusion: Swimmers have extremely short TST preceding early morning sessions and increased TST-variability with more early morning sessions.
\end{abstract}

Keywords: early morning training, intraindividual variability, swimming

According to the National Sleep Foundation, teenagers are recommended to sleep for 8 to 10 hours each night (13). Inadequate sleep duration is believed to have adverse effects on general health and well-being in young people (31), and a negative effect on athletic performance and recovery $(10,30)$. On the other hand, exercise and athletic training may also affect sleep quality and duration (15), and thus, the relationship between sleep and exercise is complex and reciprocal (7).

Besides average sleep duration, consistency of sleep patterns (eg, bedtime and sleep duration on weekdays vs weekends or nightto-night variability) may also affect restitution and health as intraindividual variability in sleep has been related to a number of physical and neurodevelopmental conditions in children and adolescents, as well as psychopathology symptoms, weight, stress, cognitive functioning, and poorer sleep habits (2). Intraindividual variability of sleep duration and sleep quality has been found to have a stronger impact on adults' well-being than average sleep duration (20).

It has been argued that adult elite athletes may need more sleep than the recommended 7 to 9 hours per night for adults to meet sustained training loads with optimum health (6). However, previous studies have found that adult elite athletes tend to have insufficient sleep duration $(19,28)$. Sleep patterns seem to differ across athletic disciplines as athletes participating in individual sports have been reported to sleep less than team sport athletes (17). Early morning training sessions $(\leq 6$ AM) may have negative effects on sleep duration. Among adults, total sleep time (TST) has been found to be less than 5 hours for sessions starting between 5 and 6 AM, but increasing to over 7 hours for sessions starting between 10 and 11 AM (28).

Only a few publications have reported on sleep in young, but not necessarily elite, athletes. Beltran-Valls et al (4) found no

The author is with the Center for Sport and Health Science, School of Education, University of Iceland, Reykjavik, Iceland. Gudmundsdottir (slg@hi.is) is corresponding author. differences in sleep quality or duration when comparing young (mean age 13.9 [0.3] y) athletes who competed regularly in organized sport events and trained $\geq 3$ days per week with nonathletes or inactive adolescents, and Brand et al. (5) found no difference in self-reported sleep duration between adolescent athletes and controls, although athletes reported better sleep quality and fewer awakenings. Interestingly, in a recent study of adolescent athletes, $4 \%-11 \%$ reported clinically relevant insomnia symptoms (11). The International Olympic Committee's Medical Commission recommends adequate sleep as part of a balanced lifestyle for elite child athletes (23). With a scarce literature on the subject, one can only speculate that as sleep deprivation is commonly found among adult elite athletes, it may also be frequent in younger elite athletes. Elite athletic training with high training loads may begin at a young age when growth and maturation are still ongoing, and social and academic demands of adolescence are being dealt with as pointed out by the Medical Commission (23). Swimming is known to impose hard training loads on athletes from young age with early morning sessions being scheduled for children and young adolescents when training in the evenings is no longer enough to match rivals' performance (12). Whitworth-Turner et al (39) reported longer sleep duration but lowered sleep efficiency and increased intraindividual night-to-night variability of sleep in young male soccer players $(n=12)$ compared with nonathlete controls. The young soccer players had training sessions starting at 10:30 AM, but morning training sessions in the sport of swimming are typically scheduled at 5:30 to $6 \mathrm{AM}$ in order for young swimmers to be able to attend school (12). Addressing intraindividual variability in athletes' sleep patterns may elucidate the importance of sport-specific and even individualized sleep hygiene education and interventions aimed at promoting sleep (9).

The aim of this study was to objectively investigate sleep duration and intraindividual night-to-night variability of sleep duration as well as wakening after sleep onset (WASO) and sleep efficiency in young Icelandic swimmers, to compare those sleep characteristics during nights preceding early morning practices with 
other nights, and to investigate sleep characteristics of nights following a day with early morning only, evening only, or both a morning and an evening session. It was hypothesized that TST would be shorter on nights preceding early morning training sessions compared with nights preceding later or no training sessions and that swimmers participating in early morning trainings would have increased night-to-night variability in sleep compared with those who did not participate in early morning training sessions. Furthermore, it was hypothesized that TST would be increased following days with both morning and evening training sessions, compared with days with either no or one training session.

\section{Methods}

\section{Setting}

All swimmers registered in the best group within the 6 largest swimming clubs in Iceland were invited to participate in the study $(n=130)$. Data were collected during a peak training period before a reduction in training (tapering) before the Icelandic championships started.

Ethical approval was granted by the ethical committee of the University of Iceland. This study was conducted according to the guidelines laid down in the Declaration of Helsinki. Parents of participants younger than 18 years of age and participants themselves when 18 or older signed an informed consent form.

\section{Sleep Monitors}

Sleep was measured with wrist-worn accelerometers (ActiGraph GT3X+, ActiSleep by ActiGraph Inc, Pensacola, FL). All accelerometers were donned by the same researcher during a training session at each club, during a single week (April 4-9, 2016). The watch-like meters were placed on the nondominant wrist of each subject and worn continuously for 7 days. Raw triaxial data were sampled at 80 samples/second (in hertz). The ActiLife software from ActiGraph (version 6.13.0.) was used to obtain rest and sleep durations, and sleep quality parameters, by use of a sleep detection algorithm validated for adolescents (26). Thus, data collection and detection correspond to previous studies of sleep in Icelandic adolescents (24). Activity monitors have been validated against polysomnography, which is considered the gold standard of sleep measurements, in young elite athletes during periods of hard training, indicating agreement rates for different sleep-wake thresholds of $81 \%-90 \%$ (29).

\section{Sleep Parameters}

To estimate the sleep duration of participants, TST was obtained, in addition to estimation of the total time spent in bed, total rest time. The SD of TST for each individual was used to describe withinsubject night-to-night variability (in minutes). WASO was used for estimation of sleep quality. WASO refers to periods (in minutes) of wakefulness occurring after sleep onset. Sleep efficiency was calculated as minutes of total sleep divided by the total time in bed and multiplied by 100 (32).

\section{Sleep and Training Logs}

Athletes filled out sleep and training logs where they registered clock times of going to bed, time of falling asleep, wake-up times, and time out of bed as well as the timing of practices during the day. Sleep logs were used to confirm the accelerometer-detected rest periods and make minor adjustments where needed. Training logs were used to confirm activity detected by the accelerometers.

\section{Body Mass Index}

Measures of standing height and body weight were performed at individual swimming pools with subjects wearing swimwear. Standing height (in meters), measured to the nearest millimeter with a stadiometer (model 217; Seca Ltd, Birmingham, UK), and body weight (in kilograms), measured to the nearest $0.1 \mathrm{~kg}$ on a calibrated scale (model 813; Seca Ltd, Birmingham, UK), were used to calculate participants' body mass index (BMI, in kilograms per meters squared).

\section{Classification of Nights and Training Days}

Each night was coded both (1) with regard to the training schedule on the following day and (2) with regard to the training schedule on the preceding day. Thus, with regard to the following day, nights were classified into 3 categories: night preceding early morning practice $(<7 \mathrm{AM})$, night preceding later $(\geq 7 \mathrm{AM})$ morning practice, and night preceding no morning practice. With regard to training on the preceding day, each night was classified into 1 of 4 categories: night following an early morning practice only, night following morning and evening practice, night following an evening practice only, and night following a day without practice.

\section{Statistical Methods}

Statistical analyses were completed using SPSS (version 24.0.; IBM SPSS Statistics, Armonk, NY). Normal distribution of continuous variables was tested with the Kolmogorov-Smirnov normality test. Descriptive summaries are presented as means and SDs. Differences between sexes and age groups were evaluated by unpaired $t$ tests. A general linear model univariate analysis was used to assess the relationship between the total number of early morning practices during the week for each subject and mean TST for the week, adjusting for age and sex. General linear model univariate analysis was also used to assess the relationship between number of early morning practices during the week and intraindividual variability in TST (TST-variability), adjusting for age, sex, and sleep time as recommended in analyses of sleep variability (2). This approach, however, does not account for the repeated measures obtained for each individual. Thus, mixed models were run separately for assessing (1) the effect of the following morning training (early, late, and none) and (2) the effect of the preceding day training (only early morning training, only evening training, 2 training sessions, and no training) on each dependent variable (TST, WASO, and efficiency) using linear mixed effects models. In each model, (1) the following morning training (early, late, and none) and (2) the effect of the preceding day training (only early morning training, only evening training, 2 training sessions, and no training) were specified as a fixed effect and entered as a repeated variable. To account for dependency in each model, participants were specified as a random effect. Effect sizes were calculated as partial eta squared $\left(\eta_{\mathrm{p}}^{2}\right)$ to assess the practical significance of the findings. Effect sizes of $<0.06$ are regarded as small $(\mathrm{S})$, moderate effect sizes range from 0.06 to $0.14(\mathrm{M})$, and large effect sizes are $>0.14$ (L) (8).

A visual inspection of a scatter plot indicated that sleep duration was correlated with age up to the age of 16 years, after which the association seemed to attenuate. This was confirmed with Pearson correlation $(P<.001$ for $<16$ y old and $P=.71$ 
for $\geq 16$ y old). The sample was, hence, stratified into 2 age groups: under the age of 16 years, and 16 years and older. This decision was further supported by the change from secondary school to high school happening at the age of 16 in Iceland with corresponding adjustments in social and academic settings. Analyses were adjusted for the potential confounding effects of age, sex, and body mass index. Significant differences or relations were accepted at $\alpha<.05$.

\section{Results}

A total of 112 swimmers (86.2\% participation rate) participated in the study. Three withdrew due to discomfort of wearing the meters during practice and sleep, and 1 meter was damaged during the study and did not return usable data. Eight participants had missing data for 1 night and parts of daytime within the data collection (weekly mean calculations are based on 6 nights for those 8 participants). Thus, sleep data were available from 108 swimmers for a total of 748 nights.

Participants' characteristics are presented by sex in Table 1. Continuous variables fit acceptably to a normal distribution. The age of the participants ranged from 10.5 to 24.0 years. The shortest mean TST for the whole week was 5 hours and 2 minutes, whereas the longest one was 8 hours and 30 minutes.

The age- and sex-adjusted association between number of early morning practices and TST is shown in Figure 1. A total of 27 swimmers did not participate in any early morning practice during the week, whereas $20,18,32$, and 11 swimmers participated in 1 , 2,3 , and 4 or more early practices, respectively. Those who did not participate in early morning practices had a significantly longer TST than those who participated in 3 early morning practices $(P<.05)$. Those who participated in 1 early morning practice had a significantly longer average TST than those who participated in 3, and 4 or more early practices $\left(P<.01 ; \eta_{\mathrm{p}}^{2}=.13[\mathrm{M}]\right.$ for main effects). Figure 2 shows the association between number of early morning practices and intraindividual TST-variability. Those who did not participate in early morning practices had significantly lower TST-variability than those who participated in any number of early morning practices $(P<.01)$ in analyses adjusted for age, sex, and $\operatorname{TST}\left(\eta_{\mathrm{p}}^{2}=.13[\mathrm{M}]\right.$ for main effects).
Table 2 shows gender- and body mass index-adjusted results from mixed model analyses showing TST and WASO in $<16$ and $\geq 16$ years old, by number of practices in the preceding day and by occurrence of early, late, or no morning practice the following morning. In the younger age group, TST was shortest when no practice had been performed during the preceding day, compared with when the preceding day included only early morning practice, only later practice, or both early morning and evening practice $\left(\eta_{\mathrm{p}}^{2}=.05[\mathrm{~S}]\right)$. TST was longer following days with both early morning practice and evening practice compared with TST following days with no training in $\geq 16$ years old $\left(\eta_{\mathrm{p}}^{2}=.01[\mathrm{~S}]\right)$. Among the older age group, WASO was lowest when the preceding day included both early morning and evening practices $\left(\eta_{\mathrm{p}}^{2}=.01[\mathrm{~S}]\right.$ for $<16$ years old and $\eta_{\mathrm{p}}^{2}=.04$ [S] for $\geq 16$ years old $)$.

When TST was compared across categories of early morning practice in the following morning (none, early, and late), the shortest TST was observed when swimmers had early morning

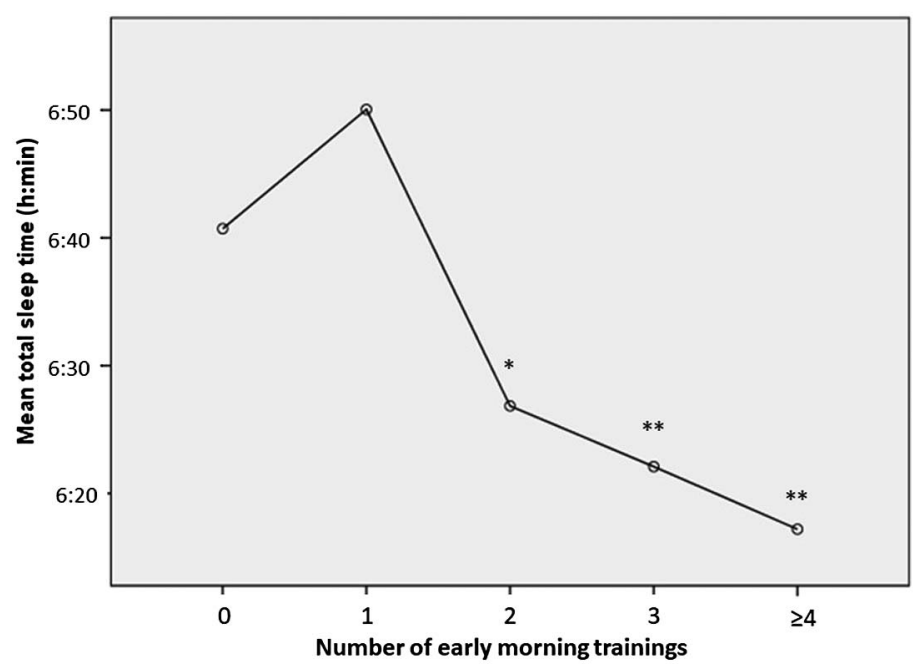

Figure 1 - Mean total sleep time over the week by number of early morning practices. Adjusted for age and sex. ${ }^{*} P<.05$ for comparison with no early morning practice. ${ }^{*} P<.01$ for comparison with 1 early morning practice.

\section{Table 1 Characteristics of Study Participants in Means (SDs) for Continuous Variables and $\mathbf{n}(\%)$ for Categorical Variables}

\begin{tabular}{|c|c|c|c|c|c|}
\hline Characteristic & All $(N=108)$ & Females $(n=69)$ & Males $(n=39)$ & $<16$ y $(n=61)$ & $\geq 16$ y $(n=47)$ \\
\hline Age, y & $16.1(2.6)$ & $15.8(2.4)$ & $16.6(2.8)$ & $14.4(1.2)$ & $18.2(2.3)^{* * *}$ \\
\hline Height, $\mathrm{cm}$ & $171.8(10.4)$ & $167.3(6.2)$ & $179.8(11.4)^{*}$ & $168.3(9.8)$ & $176.2(9.4)^{* * *}$ \\
\hline Weight, kg & $63.9(12.9)$ & $59.7(8.5)$ & $71.4(15.8)^{*}$ & $59.5(10.1)$ & $69.6(13.9)^{* * * *}$ \\
\hline BMI, $\mathrm{kg} / \mathrm{m}^{2}$ & $21.5(2.7)$ & $21.3(2.3)$ & $21.9(3.2)$ & $20.9(2.5)$ & $22.3(2.8)^{* * * *}$ \\
\hline TST, h:min & $6: 32(0: 39)$ & $6: 41(0: 35)$ & $6: 16(0: 40)^{* *}$ & $6: 46(0: 38)$ & $6: 14(0: 33)^{* * *}$ \\
\hline TRT, h:min & $7: 38(0: 42)$ & $7: 42(0: 39)$ & $7: 29(0: 45)$ & 7:51 (0:39) & $7: 21(0: 39)^{* * *}$ \\
\hline TST variability, min & $63.6(24.9)$ & $64.6(23.5)$ & $61.8(27.4)$ & $60.4(23.1)$ & $67.8(26.7)$ \\
\hline WASO, min & $63.8(24.8)$ & $59.3(21.3)$ & $71.9(28.7)^{* *}$ & $63.2(23.1)$ & $64.7(27.1)$ \\
\hline Efficiency, \% & $85.8(5.0)$ & $86.9(4.1)$ & $83.7(5.8)^{* *}$ & $86.3(4.6)$ & $85.1(5.4)$ \\
\hline Training sessions/week & $8.3(1.4)$ & $8.2(1.2)$ & $8.6(1.7)$ & $8.3(1.3)$ & $8.4(1.5)$ \\
\hline Two sessions (no. of days) & $1.5(1.2)$ & $1.4(1.0)$ & $1.8(1.4)$ & $1.4(1.1)$ & $1.7(1.2)$ \\
\hline Early morning practice (no. of days) & $1.8(1.4)$ & $1.8(1.4)$ & $1.8(1.4)$ & $1.9(1.4)$ & $1.8(1.4)$ \\
\hline
\end{tabular}

Abbreviations: BMI, body mass index; TRT, total rest time; TST, total sleep time; WASO, wake after sleep onset.

$* P<.001$ and $* * P<.05$ for differences between the sexes. $* * * P<.001$ and $* * * * P<.05$ for differences between age groups. 
Table 2 TST and WASO by Number of Training Sessions the Preceding Day and by Occurrence of Early, Late, or No Morning Session the Following Morning

\begin{tabular}{|c|c|c|c|c|}
\hline \multirow[b]{2}{*}{ Measure } & \multicolumn{2}{|c|}{ TST, h:min } & \multicolumn{2}{|c|}{ WASO, min/night } \\
\hline & $<16$ y & $\geq 16 y$ & $<16 y$ & $\geq 16 y$ \\
\hline \multicolumn{5}{|l|}{ Preceding day training } \\
\hline No training & $6: 06(5: 50-6: 22)$ & $5: 57(5: 39-6: 15)$ & $63.5(56.4-70.7)$ & $67.4(58.9-75.8)$ \\
\hline Only early morning & $6: 52(6: 19-7: 27)^{*}$ & $6: 24(5: 25-7: 23)$ & $58.8(42.9-74.2)$ & $83.4(55.9-110.8)$ \\
\hline Only late morning or evening & $6: 47(6: 38-6: 58)^{* *}$ & $6: 10(5: 58-6: 22)$ & $67.4(62.7-72.1)$ & $70.464 .7-76.2)$ \\
\hline Early morning and evening & $6: 55(6: 41-7: 09)^{* *}$ & $6: 27(6: 11-6: 45)^{*}$ & $60.1(53.6-66.5)$ & $54.4(46.6-62.2)^{*,+, \#}$ \\
\hline \multicolumn{5}{|c|}{ Morning training in following morning } \\
\hline None & 7:07 (6:58-7:15) & $6: 39$ (6:29-6:47) & $69.6(65.4-73.8)$ & $74.0(69.1-78.9)$ \\
\hline Early & $5: 36(5: 28-5: 51) * * *$ & $5: 06(4: 51-5: 20)^{* * *}$ & $54.1(48.0-60.3)^{* * *}$ & $52.7(45.2-60.3)^{* * *}$ \\
\hline Late & $6: 48(6: 30-7: 04)^{* * * *, \dagger}$ & $6: 26(6: 03-6: 47)^{\dagger}$ & $60.8(52.0-69.7)$ & $49.4(37.7-61.2)^{* * *}$ \\
\hline
\end{tabular}

Abbreviations: TST, total sleep time; WASO, wake after sleep onset. Note: Results from mixed models analyses for repeated analyses, adjusted for gender and body mass index.

$* P<.05$ and $* * P<.001$ for comparison with no training in the preceding day. $* * * P<.001$ and $* * * * P<.05$ for comparison with no morning training in the following morning. ${ }^{\dagger} P<.001$ for comparison with early morning training in the following morning. ${ }^{\ddagger} P<.05$ for comparison with only early morning training in the preceding day.

${ }^{\#} P<.01$ for comparison with only late or evening training in the preceding day.

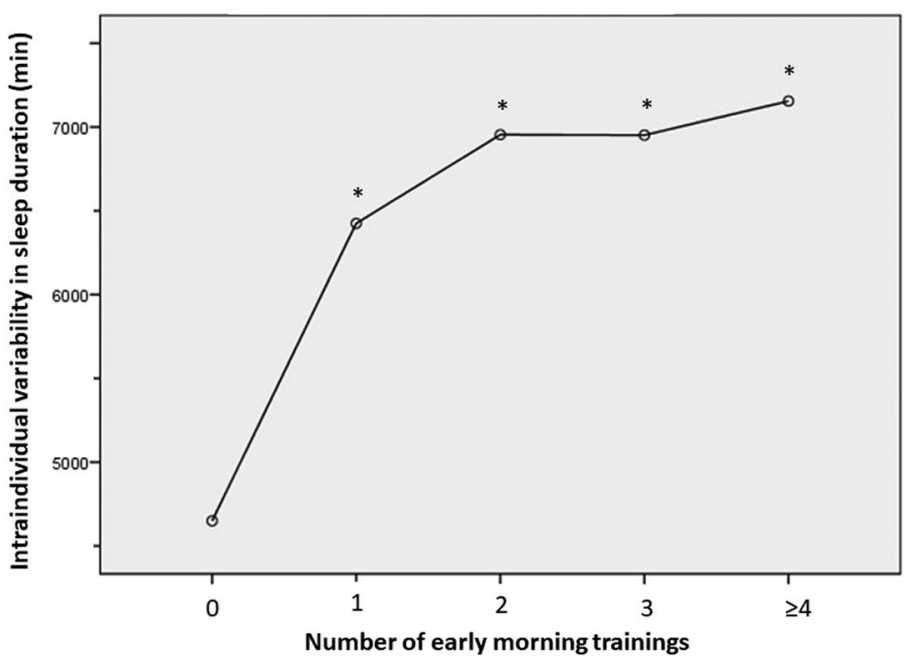

Figure 2 - Mean intraindividual variability in sleep time over the week by number of early morning practices. Adjusted for age, sex, and mean sleep time. ${ }^{*} P<.001$ for comparison with no early morning practice.

practice the following morning, both when compared with later morning practice and no morning practice $\left(\eta_{\mathrm{p}}^{2}=.29[\mathrm{~L}]\right.$ for $<16$ years old and $\eta_{\mathrm{p}}^{2}=.30$ [L] for $\geq 16$ years old). WASO was highest when there was no morning practice in the following morning $\left(\eta_{\mathrm{p}}^{2}=0.04[\mathrm{~S}]\right.$ for $<16$ years old and $\eta_{\mathrm{p}}^{2}=.11[\mathrm{M}]$ for $\geq 16$ years old $)$. Sleep efficiency did not differ across training schedules in the preceding or the following day for $<16$-year-old swimmers. For those $\geq 16$ years old, there was no difference across categories of early morning practice in the following morning but when comparing nights across categories of preceding day training, efficiency was higher when 2 training sessions had been performed $(87.6 \%)$ compared with no training $(84.0 \%)\left(P<.001\right.$ and $\left.\eta_{\mathrm{p}}^{2}=.05[\mathrm{~S}]\right)$.

\section{Discussion}

In this study, adolescent swimmers were found to have short average sleep during a whole week of measurements and significantly shorter TST on nights preceding early morning training sessions compared with nights when late or no morning training was performed. Sleep duration was shortest after days when no training was performed. Weekly mean TST decreased and intraindividual TST-variability increased as number of morning practice sessions increased. WASO was reduced during nights following days of 2 training sessions, but only in athletes aged 16 years and older.

Previous studies on sleep habits of Icelandic adolescents have repeatedly shown short sleep duration $(24,37)$. Based on objective measures, the mean TST in 15.9-year-old boys and girls was 6.2 hours on school days and 7.3 hours on nonschool days, with no difference found between those participating in organized sports and those who did not (25). The results of the current study indicate that adolescent swimmers have shorter sleep duration than nonathletic Icelandic adolescents. Findings here are consistent with the hypothesis that TST would be shortened on nights preceding early morning training. Compared with roughly 5 , and 5 and a half hours of sleep in the older and younger swimmers, respectively, TST was about 70 minutes longer when morning training sessions started later ( $\geq 7 \mathrm{AM}$ ) and 90 minutes longer when no morning training was performed. However, the hypothesis of TST being longer on nights following days with 2 training sessions compared with other days was not confirmed. TST was longest on nights following 2 session days but the difference was only significant in comparison with days without any training, and the effect size was small. Athletes have reported better sleep quality and fewer awakenings than controls (5), a difference that has later been confirmed with objective measures (16). On the other hand, it has also been suggested that disturbances in sleep are an important symptom of overreaching or overtraining in athletes, as a result of increased training load or changes in training schedule (18). Compared with a general Icelandic sample, the swimmers have a slightly higher number of WASO minutes and lower sleep efficiency (24). In particular, sleep efficiency in males was $83.7 \%$ in the current study compared with $87.5 \%$ in a previous study, whereas sleep efficiency in females in the current study was $86.9 \%$ compared with $87.8 \%$ in the general sample (25). Interestingly, WASO was lowest for the older age group after days with both an early morning session and an evening session. For both age groups, WASO was lowest during 
nights preceding early morning practice. Whether this reflects a physiologically efficient rest and restoration is unclear. The number of WASO minutes reported here is much higher than that reported by Lang et al (16) who studied a somewhat older group (16- to 25year old) of athletes, but close to the results of Suppiah et al (35) who had a similar age group as presented here but included only males from various sports disciplines. In this latter study, where the athletes had 2 training sessions during weekdays and weekend days off, sleep duration was similar to that found in this current study.

In 2015, Fullagar et al (10) published an excellent review of available research on the association between sleep and athletic performance. The effect of reduced sleep duration on athletic performance was assessed after a continuous sleep deprivation for up to 50 hours (22) or partial sleep deprivation for up to 4 nights (33). In summary, the authors found that reducing athletes' sleep for a few nights did not affect their ability to sustain maximal exercise intensity during cycling and running tests. On the other hand, decreased performances were documented in maximal work rate as well as mean and peak power produced in anaerobic cycle tests within various test populations, as well as in maximal and submaximal weightlifting tasks (10). These interventions, however, do not reflect long-term sleep deprivation or repeated weeks of one or more nights with short sleep as young swimmers may experience. Thus, it is impossible to determine whether the performance of the swimmers in the current study has suffered because of lack of sufficient sleep duration. An interesting finding by Mah et al (21), however, indicates that after extending sleep duration from a habitual nightly sleep of about 6 hours and 30 minutes to more than 8 hours, improved athletic performance was observed as well as swifter reaction time, decreased daytime sleepiness, and better mood states.

Confirming the hypothesis of increased TST-variability with participation in early morning training, intraindividual TSTvariability among the swimmers was significantly higher for any number of early morning sessions and double among swimmers participating in 4 or more early morning practices compared with those who did none. The potential effect of variable sleep patterns on athletic performance has not been studied. Highly variable sleep within individuals has been associated with a number of healthrelated outcomes in children and adolescents, particularly those related to cardiovascular health and symptoms of psychopathology such as anxiety, depression, and attention disorders (2), factors that might impair athletic performance and training ability. The overall mean TST-variability found among the swimmers is close to what Whitworth found among 19-year-old soccer players (62 min) (39). In Whitworth's study, nonathletes with a mean age of 23 years had TST-variability of 43 minutes, whereas unpublished results of a general young Icelandic sample (mean age $15.9 \mathrm{y}$ ) indicate a mean intraindividual weekly variation of TST of 75.8 minutes (unpublished data). Thus, the shift in sleep duration made by swimmers to meet training schedules may be less than the shift observed in nonathlete adolescents and is mainly explained by the shift from weekdays to weekends (40).

The mechanical pathway of how disrupted sleep may affect athletes' functioning is not clear. One of the suggested physiological effects of sleep loss on athletic performance is via disrupted cortisol function, but studies on the effects of sleep deprivation on training-induced cortisol increase in athletes are inconclusive (10). On the other hand, recent studies on the association between intraindividual variability in sleep and cortisol trajectories in adolescents and adults agree that increased intraindividual variability in sleep in adults is associated with blunted diurnal cortisol slopes and lower levels of waking cortisol $(3,38)$. As cortisol secretion in response to exercise will increase the availability of metabolic substrates during activity and recovery (1), the conceivable effect of high intraindividual sleep variability on performance and recuperation needs further study.

The data in the current study were collected in a real-life setting among a group of adolescents with a high training volume although of a quite wide range in age and athletic performance levels. Previous studies have collected data on young athletes at national level $(14,28)$ and/or during a high-intensity training period while living on a training site $(14,27)$, which may affect sleep characteristics. To the author's knowledge, only one study has previously used objective measures to assess sleep in adolescent athletes as young as those included in the current study; those were only male athletes at a high level, living in a boarding school during weekdays (35). The results of the current study indicate that concerns about inadequate sleep patterns of young athletes should not be restricted to those who have reached national or international levels, but should also cover those who may be aspiring to get there. In general, young athletes have multiple commitments, attempting to balance training and academic demands in addition to social life and, in many cases, work. School systems with flexible hours may allow for better integration of training and academic scheduling while allowing for longer sleep duration. A previous longitudinal investigation of changes in sleep patterns among Icelandic students found less reduction in sleep among students moving from primary school to a college-style scheduled secondary school than among those who moved to a traditional secondary school system (34).

The results of this study contribute to the limited knowledge on duration and regularity of sleep among adolescent athletes. A strength of the study was the use of objective, rather than self-reported, measures for sleep. Wrist-worn accelerometers have been recommended for measures of sleep parameters (41). However, activity monitors may underestimate sleep duration in individuals with larger amount of movement and higher fragmentation during sleep (29), a characteristic that has previously been found common in elite athletes $(19,36)$. The participation rate was quite high, and the study sample represents a relatively large portion of the total number of swimmers in Iceland who participate in serious training.

While training sessions may have varied in duration and intensity, only frequency and timing of sessions were recorded in this study. Two training sessions per day were only recorded if those happened at different times of the day. Yet, some sessions may have included both swimming and strength training and thus extended for longer time than the typical 1 to 2 hours. It cannot be ruled out that swimming training sessions consisting mainly of kicking with a board or strength training with predominantly leg exercises were missed, resulting in a bias in coding of the days, although training logs were used to confirm accelerometer registration. In 8 participants, the number of training sessions could be underestimated as meters indicated nonwear time of demarcated time for 2 to 4 times during the data collection. Other factors that may have affected sleep patterns such as caffeine drink consumption or screen time usage were not registered.

In conclusion, this study found that participation in early morning practices was associated with short TST as well as with increased intraindividual TST-variability in sleep among adolescent Icelandic swimmers. The difference in TST between nights preceding early morning training, and nights preceding later or no morning training was considerable. The effects of repeated bouts of sleep deprivation on competitive athletes are unknown, and the results raise concerns both about the athletes' general health 
and well-being as well as regarding the potential negative effects on their athletic performance.

\section{Acknowledgments}

The author thanks the participants of the study for their contribution as well as the coaches at the 6 participating swim clubs for their assistance. The Icelandic Sport Fund funded this study. The Icelandic Sport Fund had no role in the design, analysis, or writing of this article. The author certifies that she has no affiliations with or involvement in any organization or entity with any financial interest, or nonfinancial interest in the subject matter or materials discussed in this manuscript. S.L.G. received funding, designed and executed the study, performed the statistical analyses, and wrote the manuscript.

\section{References}

1. Anderson T, Wideman L. Exercise and the cortisol awakening response: a systematic review. Sports Med Open. 2017;3(1):37. PubMed ID: 29019089 doi:10.1186/s40798-017-0102-3

2. Becker SP, Sidol CA, Van Dyk TR, Epstein JN, Beebe DW. Intraindividual variability of sleep/wake patterns in relation to child and adolescent functioning: a systematic review. Sleep Med Rev. 2017; 34:94-121. PubMed ID: 27818086 doi:10.1016/j.smrv.2016.07.004

3. Bei B, Seeman TE, Carroll JE, Wiley JF. Sleep and physiological dysregulation: a closer look at sleep intraindividual variability. Sleep. 2017;40(9). doi:10.1093/sleep/zsx109

4. Beltran-Valls MR, Artero EG, Capdevila-Seder A, Legaz-Arrese A, Adelantado-Renau M, Moliner-Urdiales D. Regular practice of competitive sports does not impair sleep in adolescents: DADOS study. Pediatr Exerc Sci. 2018;30(2):229-36. PubMed ID: 29039246 doi:10.1123/pes.2017-0129

5. Brand S, Gerber M, Beck J, Hatzinger M, Pühse U, HolsboerTrachsler E. High exercise levels are related to favorable sleep patterns and psychological functioning in adolescents: a comparison of athletes and controls. J Adolesc Health. 2010;46(2):133-41. PubMed ID: 20113919 doi:10.1016/j.jadohealth.2009.06.018

6. Calder A. Recovery strategies for sport performance. USOC Olympic Coach E-Magazine. 2003;15(3):8-11.

7. Chennaoui M, Arnal PJ, Sauvet F, Léger D. Sleep and exercise: a reciprocal issue? Sleep Med Rev. 2015;20:59-72. PubMed ID: 25127157 doi:10.1016/j.smrv.2014.06.008

8. Cohen J. Eta-squared and partial eta-squared in communication science. Hum Commun Res. 1973;28:473-90.

9. Fullagar HH, Bartlett JD. Time to wake up: individualising the approach to sleep promotion interventions. Br J Sports Med. 2016; 50(3):143-4. PubMed ID: 26701930 doi:10.1136/bjsports-2015095759

10. Fullagar HH, Skorski S, Duffield R, Hammes D, Coutts AJ, Meyer T. Sleep and athletic performance: the effects of sleep loss on exercise performance, and physiological and cognitive responses to exercise. Sports Med. 2015;45(2):161-86. PubMed ID: 25315456 doi:10.1007/s40279-014-0260-0

11. Gerber M, Best S, Meerstetter F, et al. Cross-sectional and longitudinal associations between athlete burnout, insomnia, and polysomnographic indices in young elite athletes. J Sport Exerc Psychol. 2018; 40(6):312-24. PubMed ID: 30514157 doi:10.1123/jsep.2018-0083

12. Greyson I, Kelly S, Peyrebrune M, Furniss B. Interpreting and implementing the long term athlete development model: English swimming coaches' views on the (swimming) LTAD in practice: a commentary. Int J Sports Sci Coach. 2010;5(3):403-6. doi:10.1260/ 1747-9541.5.3.403

13. Hirshkowitz M, Whiton K, Albert SM, et al. National Sleep Foundation's updated sleep duration recommendations: final report. Sleep Health. 2015;1(4):233-43. PubMed ID: 29073398 doi:10.1016/j. sleh.2015.10.004

14. Kölling S, Steinacker JM, Endler S, Ferrauti A, Meyer T, Kellmann M. The longer the better: sleep-wake patterns during preparation of the World rowing junior championships. Chronobiol Int. 2016;33(1):73-84. doi:10.3109/07420528.2015.1118384

15. Kredlow MA, Capozzoli MC, Hearon BA, Calkins AW, Otto MW. The effects of physical activity on sleep: a meta-analytic review. $J$ Behav Med. 2015;38(3):427-49. PubMed ID: 25596964 doi:10.1007/ s10865-015-9617-6

16. Lang C, Brand S, Feldmeth AK, Holsboer-Trachsler E, Pühse U, Gerber M. Increased self-reported and objectively assessed physical activity predict sleep quality among adolescents. Physiol Behav. 2013;120:46-53. PubMed ID: 23851332 doi:10.1016/j.physbeh. 2013.07.001

17. Lastella M, Roach GD, Halson SL, Sargent C. Sleep/wake behaviours of elite athletes from individual and team sports. Eur J Sport Sci. 2015;15(2):94-100. PubMed ID: 24993935 doi:10.1080/17461391. 2014.932016

18. Lastella M, Vincent GE, Duffield R, Roach GD, Halson SL, Heales LJ, Sargent C. Can sleep be used as an indicator of overreaching and overtraining in athletes? Front Physiol. 2018;9:436. PubMed ID: 29740346 doi:10.3389/fphys.2018.00436

19. Leeder J, Glaister M, Pizzoferro K, Dawson J, Pedlar C. Sleep duration and quality in elite athletes measured using wristwatch actigraphy. J Sports Sci. 2012;30(6):541-5. PubMed ID: 22329779 doi:10.1080/02640414.2012.660188

20. Lemola S, Ledermann T, Friedman EM. Variability of sleep duration is related to subjective sleep quality and subjective well-being: an actigraphy study. PLoS ONE. 2013;8(8):e71292. PubMed ID: 23967186 doi:10.1371/journal.pone.0071292

21. Mah CD, Mah KE, Kezirian EJ, Dement WC. The effects of sleep extension on the athletic performance of collegiate basketball players. Sleep. 2011;34(7):943-50. PubMed ID: 21731144 doi:10.5665/ SLEEP. 1132

22. Martin BJ, Chen HI. Sleep loss and the sympathoadrenal response to exercise. Med Sci Sports Exerc. 1984;16(1):56-9. PubMed ID: 6423925

23. Mountjoy M, Armstrong N, Bizzini L, et al. IOC consensus statement: "Training the elite child athlete". Br J Sports Med. 2008;42(3): 163-4. PubMed ID: 18048429 doi:10.1136/bjsm.2007.044016

24. Rognvaldsdottir V, Gudmundsdottir SL, Brychta RJ, et al. Sleep deficiency on school days in Icelandic youth, as assessed by wrist accelerometry. Sleep Med. 2017;33:103-8. PubMed ID: 28449887 doi:10.1016/j.sleep.2016.12.028

25. Rognvaldsdottir V, Valdimarsdottir BM, Brychta RJ, et al. Physical activity and sleep in Icelandic adolescents. Laeknabladid. 2018; 104(2):79-85. PubMed ID: 29388918

26. Sadeh A, Sharkey KM, Carskadon MA. Activity-based sleep-wake identification: an empirical test of methodological issues. Sleep. 1994;17(3):201-7. PubMed ID: 7939118 doi:10.1093/sleep/17.3.201

27. Sargent C, Halson S, Roach GD. Sleep or swim? Early-morning training severely restricts the amount of sleep obtained by elite swimmers. Eur J Sport Sci. 2014;14 suppl 1:S310-5. doi:10.1080/ 17461391.2012.696711

28. Sargent C, Lastella M, Halson SL, Roach GD. The impact of training schedules on the sleep and fatigue of elite athletes. Chronobiol Int. 
2014;31(10):1160-8. PubMed ID: 25222347 doi:10.3109/07420528. 2014.957306

29. Sargent C, Lastella M, Halson SL, Roach GD. The validity of activity monitors for measuring sleep in elite athletes. J Sci Med Sport. 2015;19(10):848-53. PubMed ID: 26794719 doi:10.1016/j.jsams. 2015.12.007

30. Sawczuk T, Jones B, Scantlebury S, Till K. Influence of perceptions of sleep on well-being in youth athletes. J Strength Cond Res. Epub ahead of print 2018. doi:10.1519/JSC.0000000000002857

31. Shochat T, Cohen-Zion M, Tzischinsky O. Functional consequences of inadequate sleep in adolescents: a systematic review. Sleep Med Rev. 2014;18(1):75-87. PubMed ID: 23806891 doi:10.1016/j.smrv. 2013.03.005

32. Shrivastava D, Jung S, Saadat M, Sirohi R, Crewson K. How to interpret the results of a sleep study. J Community Hosp Intern Med Perspect. 2014;4(5):24983. PubMed ID: 25432643 doi:10.3402/ jchimp.v4.24983

33. Sinnerton S, Reilly T. Effects of Sleep Loss and Time of Day in Swimmers. London, UK: E and FN Spon; 1992.

34. Stefansdottir R, Rognvaldsdottir V, Chen KY, et al. Does a More Flexible School Schedule Affect Objectively Measured Sleep, Physical Activity, and Body Composition in Icelandic youth? (abstract). Health Across Lifespan; 2018. Magdeburg, Germany.

35. Suppiah HT, Low CY, Chia M. Effects of sport-specific training intensity on sleep patterns and psychomotor performance in adolescent athletes. Pediatr Exerc Sci. 2016;28(4):588-95. PubMed ID: 26757487 doi:10.1123/pes.2015-0205
36. Taylor SR, Rogers GG, Driver HS. Effects of training volume on sleep, psychological, and selected physiological profiles of elite female swimmers. Med Sci Sports Exerc. 1997;29(5):68893. PubMed ID: 9140908 doi:10.1097/00005768-19970500000016

37. Thorleifsdottir B, Bjornsson JK, Benediktsdottir B, Gislason T, Kristbjarnarson H. Sleep and sleep habits from childhood to young adulthood over a 10-year period. J Psychosom Res. 2002;53(1):52937. PubMed ID: 12127168 doi:10.1016/S0022-3999(02)00444-0

38. Van Lenten SA, Doane LD. Examining multiple sleep behaviors and diurnal salivary cortisol and alpha-amylase: within- and between-person associations. Psychoneuroendocrinology. 2016; 68:100-10. PubMed ID: 26963376 doi:10.1016/j.psyneuen.2016. 02.017

39. Whitworth-Turner C, Di Michele R, Muir I, Gregson W, Drust B. A comparison of sleep patterns in youth soccer players and non-athletes. Sci Med in Football. 2018;2(1):3-8. doi:10.1080/24733938.2017. 1366040

40. Wittmann M, Dinich J, Merrow M, Roenneberg T. Social jetlag: misalignment of biological and social time. Chronobiol Int. 2006;23(1-2):497-509. PubMed ID: 16687322 doi:10.1080/ 07420520500545979

41. Zinkhan M, Berger K, Hense S, et al. Agreement of different methods for assessing sleep characteristics: a comparison of two Actigraphs, wrist and hip placement, and self-report with polysomnography. Sleep Med. 2014;15(9):1107-14. PubMed ID: 25018025 doi:10. 1016/j.sleep.2014.04.015 